\title{
A General Delta-Nabla Calculus of Variations on Time Scales with Application to Economics*
}

\author{
Monika Dryl \\ monikadryl@ua.pt \\ Delfim F. M. Torres
delfim@ua.pt \\ Center for Research and Development in Mathematics and Applications (CIDMA) \\ Department of Mathematics, University of Aveiro, 3810-193 Aveiro, Portugal
}

\begin{abstract}
We consider a general problem of the calculus of variations on time scales with a cost functional that is the composition of a certain scalar function with delta and nabla integrals of a vector valued field. Euler-Lagrange delta-nabla differential equations are proved, which lead to important insights in the process of discretization. Application of the obtained results to a firm that wants to program its production and investment policies to reach a given production rate and to maximize its future market competitiveness is discussed.
\end{abstract}

Keywords: time scales, calculus of variations, Euler-Lagrange equations, discretizations, application to economics.

2010 Mathematics Subject Classification: 34N05; 49K05; 91B02; 91B62.

\section{Introduction}

The calculus of variations on time scales has been developing rapidly in the past nine years, after the pioneering work [6], and is now a fertile area of research. Indeed, in order to deal with nontraditional applications in economics, where the system dynamics are described on a time scale partly continuous and partly discrete, or to accommodate nonuniform sampled systems, one needs to work with variational problems defined on a time scale [3, 5, 13. Here we study general nonclassical problems of the calculus of variations on time scales. More precisely, we consider the problem of minimizing or maximizing a composition of delta and nabla integral functionals. Main results include new necessary optimality conditions (Theorem 3.2) that lead to better discretizations with relevance in economics.

The paper is organized as follows. In Section 2 we collect the necessary background on the nabla and delta calculus on time scales. In Section 3 we formulate the general (nonclassical) mixed delta-nabla problem (5) -(6) of the calculus of variations on time scales. We prove general necessary optimality conditions of Euler-Lagrange type in differential form (Theorem 3.2), which are then applied to the particular time scales $\mathbb{T}=\mathbb{R}$ (Corollary 3.1) and $\mathbb{T}=\mathbb{Z}$ (Corollary 3.2). In Section 4 we consider an economic problem describing a firm that wants to program its production and investment policies to reach a given production rate and to maximize its future market competitiveness. The continuous case, denoted by $(P)$, was discussed in [11; here we focus our attention on different discretizations of problem $(P)$, in particular to the mixed delta-nabla discretizations that we call $\left(P_{\Delta \nabla}\right)$ and $\left(P_{\nabla \Delta}\right)$. For these discrete problems the direct discretization

*This is a preprint of a paper whose final and definite form will be published in the Int. J. Dyn. Syst. Differ. Equ. (IJDSDE), ISSN 1752-3583. Paper submitted 17/Jul/2014; revised 21/Sept/2014 and 03/Oct/2014; accepted for publication 05/Oct/2014. Part of first author's Ph.D., carried out at the University of Aveiro under the Doctoral Programme in Mathematics and Applications of Universities of Aveiro and Minho. 
of the Euler-Lagrange equation for $(P)$ does not lead to the solution of the problems: the results found by applying our Corollary 3.2 to $\left(P_{\Delta \nabla}\right)$ and $\left(P_{\nabla \Delta}\right)$ are shown to be better. The comparison is done in Section 5 . We end with Section 6 of conclusion and future work.

\section{Preliminaries}

In this section we review some basic definitions and theorems that are useful in the sequel. For more details concerning the theory of time scales we refer to the books 8,9 . For the calculus of variations on time scales see [18, 22, 26] and references therein. All the intervals in this paper are time scale intervals.

Definition 2.1. A time scale $\mathbb{T}$ is an arbitrary nonempty closed subset of $\mathbb{R}$. Given a time scale $\mathbb{T}$, the backward jump operator $\rho: \mathbb{T} \rightarrow \mathbb{T}$ is defined by $\rho(t):=\sup \{s \in \mathbb{T}: s<t\}$ for $t \neq \inf \mathbb{T}$ and $\rho(\inf \mathbb{T}):=\inf \mathbb{T}$ if inf $\mathbb{T}>-\infty$. The forward jump operator $\sigma: \mathbb{T} \rightarrow \mathbb{T}$ is defined by $\sigma(t):=\inf \{s \in \mathbb{T}: s>t\}$ for $t \neq \sup \mathbb{T}$ and $\sigma(\sup \mathbb{T}):=\sup \mathbb{T}$ if $\sup \mathbb{T}<+\infty$.

A point $t \in \mathbb{T}$ is right-dense or right-scattered, left-dense or left-scattered, if $\sigma(t)=t$ or $\sigma(t)>t$, $\rho(t)=t$ or $\rho(t)<t$, respectively.

Definition 2.2. The backward graininess function $\nu: \mathbb{T} \rightarrow[0, \infty)$ is defined by $\nu(t):=t-\rho(t)$; the forward graininess function $\mu: \mathbb{T} \rightarrow[0, \infty)$ is defined by $\mu(t):=\sigma(t)-t$.

Example 2.1. If $\mathbb{T}=h \mathbb{Z}, h>0$, then $\sigma(t)=t+h, \rho(t)=t-h$, and $\mu(t)=\nu(t) \equiv h$.

To simplify the notation, we use $f^{\rho}(t):=f(\rho(t))$ and $f^{\sigma}(t):=f(\sigma(t))$. If $\mathbb{T}$ has a rightscattered minimum $m$, then we define $\mathbb{T}_{\kappa}:=\mathbb{T}-\{m\}$; otherwise, we set $\mathbb{T}_{\kappa}:=\mathbb{T}$. Similarly, if $\sup \mathbb{T}$ is finite and left-scattered, then we define $\mathbb{T}^{\kappa}:=\mathbb{T}-\{\sup \mathbb{T}\}$; otherwise, we set $\mathbb{T}^{\kappa}:=\mathbb{T}$. Let us define the sets $\mathbb{T}^{\kappa^{n}}, n \geq 2$, inductively: $\mathbb{T}^{\kappa^{1}}:=\mathbb{T}^{\kappa}$ and $\mathbb{T}^{\kappa^{n}}:=\left(\mathbb{T}^{\kappa^{n-1}}\right)^{\kappa}, n \geq 2$. Similarly, $\mathbb{T}_{\kappa^{1}}:=\mathbb{T}_{\kappa}$ and $\mathbb{T}_{\kappa^{n}}:=\left(\mathbb{T}_{\kappa^{n-1}}\right)_{\kappa}, n \geq 2$. Finally, we define $\mathbb{T}_{\kappa}^{\kappa}:=\mathbb{T}_{\kappa} \cap \mathbb{T}^{\kappa}$.

\subsection{The nabla approach to time scales}

The nabla approach is based on the $\rho$ operator.

Definition 2.3 (Section 3.1 of $[9]$ ). We say that a function $f: \mathbb{T} \rightarrow \mathbb{R}$ is nabla differentiable at $t \in \mathbb{T}_{\kappa}$ if there is a number $f^{\nabla}(t)$ such that for all $\varepsilon>0$ there exists a neighborhood $U$ of $t$ (i.e., $U=(t-\delta, t+\delta) \cap \mathbb{T}$ for some $\delta>0)$ such that

$$
\left|f^{\rho}(t)-f(s)-f^{\nabla}(t)(\rho(t)-s)\right| \leq \varepsilon|\rho(t)-s| \text { for all } s \in U .
$$

We say that $f^{\nabla}(t)$ is the nabla derivative of $f$ at $t$. Moreover, $f$ is said to be nabla differentiable on $\mathbb{T}$ provided $f^{\nabla}(t)$ exists for all $t \in \mathbb{T}_{\kappa}$.

Theorem 2.4 (Theorem 8.39 of [8]). Let $f: \mathbb{T} \rightarrow \mathbb{R}$ and $t \in \mathbb{T}_{\kappa}$. If $f$ is continuous at $t$ and $t$ is left-scattered, then $f$ is nabla differentiable at $t$ with

$$
f^{\nabla}(t)=\frac{f(t)-f(\rho(t))}{\nu(t)} .
$$

Theorem 2.5 (Theorem 8.41. of [8]). Let $f, g: \mathbb{T} \rightarrow \mathbb{R}$ be nabla differentiable at $t \in \mathbb{T}_{\kappa}$. Then,

1. the sum $f+g: \mathbb{T} \rightarrow \mathbb{R}$ is nabla differentiable at $t$ with

$$
(f+g)^{\nabla}(t)=f^{\nabla}(t)+g^{\nabla}(t)
$$

2. for any constant $\alpha, \alpha f: \mathbb{T} \rightarrow \mathbb{R}$ is nabla differentiable at $t$ with

$$
(\alpha f)^{\nabla}(t)=\alpha f^{\nabla}(t)
$$


3. the product $f g: \mathbb{T} \rightarrow \mathbb{R}$ is nabla differentiable at $t$ with

$$
(f g)^{\nabla}(t)=f^{\nabla}(t) g(t)+f^{\rho} g^{\nabla}(t)=f(t) g^{\nabla}(t)+f^{\nabla}(t) g^{\rho}(t) ;
$$

4. if $g(t) g^{\rho}(t) \neq 0$, then $\mathrm{f} / \mathrm{g}$ is nabla differentiable at $t$ with

$$
\left(\frac{f}{g}\right)^{\nabla}(t)=\frac{f^{\nabla}(t) g(t)-f(t) g^{\nabla}(t)}{g(t) g^{\rho}(t)} .
$$

Definition 2.6 (Section 3.1 of $[9]$ ). Let $\mathbb{T}$ be a time scale and $f: \mathbb{T} \rightarrow \mathbb{R}$. We say that $f$ is ld-continuous if it is continuous at left-dense points and its right-sided limits exists (finite) at all right-dense points.

The set of all ld-continuous functions $f: \mathbb{T} \rightarrow \mathbb{R}$ is denoted by

$$
C_{l d}=C_{l d}(\mathbb{T})=C_{l d}(\mathbb{T}, \mathbb{R})
$$

and the set of all nabla differentiable functions with ld-continuous derivative by

$$
C_{l d}^{1}=C_{l d}^{1}(\mathbb{T})=C_{l d}^{1}(\mathbb{T}, \mathbb{R}) .
$$

Theorem 2.7 (Theorems 8.46 and 8.47 of $[8]$ and Theorem 8 of [25]). If $a, b, c \in \mathbb{T}, a \leq c \leq b$, $\alpha \in \mathbb{R}$, and $f, g \in C_{l d}(\mathbb{T}, \mathbb{R})$, then:

1. $\int_{a}^{b}(f(t)+g(t)) \nabla t=\int_{a}^{b} f(t) \nabla t+\int_{a}^{b} g(t) \nabla t ;$

2. $\int_{a}^{b} \alpha f(t) \nabla t=\alpha \int_{a}^{b} f(t) \nabla t$

3. $\int_{a}^{b} f(t) \nabla t=\int_{a}^{c} f(t) \nabla t+\int_{c}^{b} f(t) \nabla t$;

4. $\int_{a}^{a} f(t) \nabla t=0$;

5. if $f, g \in C_{l d}^{1}(\mathbb{T}, \mathbb{R})$, then $\int_{a}^{b} f(t) g^{\nabla}(t) \nabla t=\left.f(t) g(t)\right|_{t=a} ^{t=b}-\int_{a}^{b} f^{\nabla}(t) g(\rho(t)) \nabla t$;

6. if $f(t) \geq 0$ for all $a<t \leq b$, then $\int_{a}^{b} f(t) \nabla t \geq 0$;

7. if $t \in \mathbb{T}_{\kappa}$, then $\int_{\rho(t)}^{t} f(\tau) \nabla \tau=\nu(t) f(t)$.

\subsection{The delta approach to time scales}

The delta calculus is similar to the nabla one (Section 2.1) with $\sigma$ taking the role of operator $\rho$.

Definition 2.8 (Section 1.1 of [8]). Let $f: \mathbb{T} \rightarrow \mathbb{R}$ and $t \in \mathbb{T}^{\kappa}$. We define $f^{\Delta}(t)$ to be the number (provided it exists) with the property that given any $\varepsilon>0$, there is a neighborhood $U$ of $t$ such that

$$
\left|f^{\sigma}(t)-f(s)-f^{\Delta}(t)(\sigma(t)-s)\right| \leq \varepsilon|\sigma(t)-s| \text { for all } s \in U .
$$

We call $f^{\Delta}(t)$ the delta derivative of $f$ at $t$. Function $f$ is delta differentiable on $\mathbb{T}^{\kappa}$ provided $f^{\Delta}(t)$ exists for all $t \in \mathbb{T}^{\kappa}$. Then, $f^{\Delta}: \mathbb{T}^{\kappa} \rightarrow \mathbb{R}$ is called the delta derivative of $f$ on $\mathbb{T}^{\kappa}$. 
Theorem 2.9 (Theorem 1.16 of [8]). Let $f: \mathbb{T} \rightarrow \mathbb{R}$ and $t \in \mathbb{T}^{\kappa}$. If $f$ is continuous at $t$ and $t$ is right-scattered, then $f$ is delta differentiable at $t$ with

$$
f^{\Delta}(t)=\frac{f(\sigma(t))-f(t)}{\mu(t)} .
$$

Theorem 2.10 (Theorem 1.20 of [8]). Let $f, g: \mathbb{T} \rightarrow \mathbb{R}$ be delta differentiable at $t \in \mathbb{T}^{\kappa}$. Then,

1. the sum $f+g: \mathbb{T} \rightarrow \mathbb{R}$ is delta differentiable at $t$ with

$$
(f+g)^{\Delta}(t)=f^{\Delta}(t)+g^{\Delta}(t) ;
$$

2. for any constant $\alpha, \alpha f: \mathbb{T} \rightarrow \mathbb{R}$ is delta differentiable at $t$ with

$$
(\alpha f)^{\Delta}(t)=\alpha f^{\Delta}(t)
$$

3. the product $f g: \mathbb{T} \rightarrow \mathbb{R}$ is delta differentiable at $t$ with

$$
(f g)^{\Delta}(t)=f^{\Delta}(t) g(t)+f^{\sigma} g^{\Delta}(t)=f(t) g^{\Delta}(t)+f^{\Delta}(t) g^{\sigma}(t) ;
$$

4. if $g(t) g^{\sigma}(t) \neq 0$, then $f / g$ is delta differentiable at $t$ with

$$
\left(\frac{f}{g}\right)^{\Delta}(t)=\frac{f^{\Delta}(t) g(t)-f(t) g^{\Delta}(t)}{g(t) g^{\sigma}(t)} .
$$

Definition 2.11 (Section 1.4 of $[9$ ). A function $f: \mathbb{T} \rightarrow \mathbb{R}$ is called rd-continuous provided it is continuous at right-dense points in $\mathbb{T}$ and its left-sided limits exist (finite) at all left-dense points in $\mathbb{T}$.

The set of all rd-continuous functions $f: \mathbb{T} \rightarrow \mathbb{R}$ is denoted by

$$
C_{r d}=C_{r d}(\mathbb{T})=C_{r d}(\mathbb{T}, \mathbb{R}) .
$$

The set of functions $f: \mathbb{T} \rightarrow \mathbb{R}$ that are delta differentiable and whose derivative is rd-continuous is denoted by

$$
C_{r d}^{1}=C_{r d}^{1}(\mathbb{T})=C_{r d}^{1}(\mathbb{T}, \mathbb{R}) .
$$

Theorem 2.12 (Theorems 1.75 and 1.77 of []). If $a, b, c \in \mathbb{T}, a \leq c \leq b, \alpha \in \mathbb{R}$, and $f, g \in$ $C_{r d}(\mathbb{T}, \mathbb{R})$, then

1. $\int_{a}^{b}(f(t)+g(t)) \Delta t=\int_{a}^{b} f(t) \Delta t+\int_{a}^{b} g(t) \Delta t$

2. $\int_{a}^{b} \alpha f(t) \Delta t=\alpha \int_{a}^{b} f(t) \Delta t$

3. $\int_{a}^{b} f(t) \Delta t=\int_{a}^{c} f(t) \Delta t+\int_{c}^{b} f(t) \Delta t$

4. $\int_{a}^{a} f(t) \Delta t=0$

5. if $f, g \in C_{r d}^{1}(\mathbb{T}, \mathbb{R})$, then $\int_{a}^{b} f(t) g^{\Delta}(t) \Delta t=\left.f(t) g(t)\right|_{t=a} ^{t=b}-\int_{a}^{b} f^{\Delta}(t) g(\sigma(t)) \Delta t$;

6. if $f, g \in C_{r d}^{1}(\mathbb{T}, \mathbb{R})$, then $\int_{a}^{b} f(\sigma(t)) g^{\Delta}(t) \Delta t=\left.f(t) g(t)\right|_{t=a} ^{t=b}-\int_{a}^{b} f^{\Delta}(t) g(t) \Delta t$;

7. if $f(t) \geq 0$ for all $a \leq t<b$, then $\int_{a}^{b} f(t) \Delta t \geq 0$;

8. if $t \in \mathbb{T}^{\kappa}$, then $\int_{t}^{\sigma(t)} f(\tau) \Delta \tau=\mu(t) f(t)$. 


\subsection{Relation between delta and nabla approaches to time-scale calculus}

It is possible to relate the approach of Section 2.1 with that of Section 2.2 .

Theorem 2.13 (Theorems 2.5 and 2.6 of [4]). If $f: \mathbb{T} \rightarrow \mathbb{R}$ is delta differentiable on $\mathbb{T}^{\kappa}$ and $f^{\Delta}$ is continuous on $\mathbb{T}^{\kappa}$, then $f$ is nabla differentiable on $\mathbb{T}_{\kappa}$ with

$$
f^{\nabla}(t)=\left(f^{\Delta}\right)^{\rho}(t) \text { for all } t \in \mathbb{T}_{\kappa} .
$$

If $f: \mathbb{T} \rightarrow \mathbb{R}$ is nabla differentiable on $\mathbb{T}_{\kappa}$ and $f^{\nabla}$ is continuous on $\mathbb{T}_{\kappa}$, then $f$ is delta differentiable on $\mathbb{T}^{\kappa}$ with

$$
f^{\Delta}(t)=\left(f^{\nabla}\right)^{\sigma}(t) \text { for all } t \in \mathbb{T}^{\kappa} .
$$

Theorem 2.14 (Proposition 7 of $[20]$ ). If function $f: \mathbb{T} \rightarrow \mathbb{R}$ is continuous, then for all $a, b \in \mathbb{T}$ with $a<b$ we have

$$
\begin{aligned}
& \int_{a}^{b} f(t) \Delta t=\int_{a}^{b} f^{\rho}(t) \nabla t, \\
& \int_{a}^{b} f(t) \nabla t=\int_{a}^{b} f^{\sigma}(t) \Delta t .
\end{aligned}
$$

For a different approach relating the delta and the nabla calculi, based on duality, we refer the reader to 10,19 .

\section{Main results}

By $\mathcal{C}^{1}$ we denote the class of continuous functions $y:[a, b] \rightarrow \mathbb{R}$ that are simultaneously delta and nabla differentiable with $y^{\Delta}(t)$ and $y^{\nabla}(t)$ continuous on $[a, b]_{\kappa}^{\kappa}$. Let $k, n \in \mathbb{N}=\{1,2, \ldots\}$, let $\mathbb{T}$ be a given time scale with at least three points, and let $a, b \in \mathbb{T}$. We consider the following general problem of the calculus of variations on time scales.

Problem. Find a function $y \in \mathcal{C}^{1}$ that extremizes (minimizes or maximizes) the functional

$$
\begin{aligned}
\mathcal{L}[y]=H\left(\int_{a}^{b} f_{1}\left(t, y^{\sigma}(t), y^{\Delta}(t)\right) \Delta t, \ldots, \int_{a}^{b} f_{k}\left(t, y^{\sigma}(t), y^{\Delta}(t)\right) \Delta t\right. & \\
& \left.\int_{a}^{b} f_{k+1}\left(t, y^{\rho}(t), y^{\nabla}(t)\right) \nabla t, \ldots, \int_{a}^{b} f_{k+n}\left(t, y^{\rho}(t), y^{\nabla}(t)\right) \nabla t\right)
\end{aligned}
$$

subject to the boundary conditions

$$
y(a)=y_{a}, \quad y(b)=y_{b},
$$

and under the assumptions that

1. function $H: \mathbb{R}^{n+k} \rightarrow \mathbb{R}$ has continuous partial derivatives with respect to its arguments, which we denote by $H_{i}^{\prime}, i=1, \ldots, n+k$;

2. functions $(t, y, v) \rightarrow f_{i}(t, y, v)$ from $[a, b] \times \mathbb{R}^{2}$ to $\mathbb{R}, i=1, \ldots, n+k$, have continuous partial derivatives with respect to $y$ and $v$ uniformly in $t \in[a, b]$, which we denote by $f_{i y}$ and $f_{i v}$, respectively;

3. functions $f_{i}, f_{i y}, f_{i v}$ are rd-continuous in $t \in[a, b]^{\kappa}, i=1, \ldots, k$, and ld-continuous in $t \in[a, b]_{\kappa}, i=k+1, \ldots, k+n$, for all $y \in \mathcal{C}^{1}$. 
A function $y \in \mathcal{C}^{1}$ is said to be admissible provided it satisfies the boundary conditions (6). In order to introduce the notion of solution to our problem, we consider the following norm in $\mathcal{C}^{1}$ :

$$
\|y\|_{1, \infty}:=\left\|y^{\sigma}\right\|_{\infty}+\left\|y^{\Delta}\right\|_{\infty}+\left\|y^{\rho}\right\|_{\infty}+\left\|y^{\nabla}\right\|_{\infty},
$$

where $\|y\|_{\infty}:=\sup _{t \in[a, b] \cap \mathbb{T}_{\kappa}^{\kappa}}|y(t)|$.

Definition 3.1. We say that an admissible function $\hat{y}$ is a local minimizer (respectively, local maximizer) to problem (5) -(6) if there exists $\delta>0$ such that $\mathcal{L}[\hat{y}] \leq \mathcal{L}[y]$ (respectively, $\mathcal{L}[\hat{y}] \geq \mathcal{L}[y]$ ) for all admissible functions $y \in \mathcal{C}^{1}$ satisfying the inequality $\|y-\hat{y}\|_{1, \infty}<\delta$.

For brevity, in what follows we omit the argument of $H_{i}^{\prime}$. Precisely, $H_{i}^{\prime}:=H_{i}^{\prime}\left(\mathcal{F}_{1}[\hat{y}], \ldots, \mathcal{F}_{k+n}[\hat{y}]\right)$, where $\mathcal{F}_{i}[\hat{y}]:=\int_{a}^{b} f_{i}\left(t, \hat{y}^{\sigma}(t), \hat{y}^{\Delta}(t)\right) \Delta t$ for $i=1, \ldots, k$ and $\mathcal{F}_{i}[\hat{y}]:=\int_{a}^{b} f_{i}\left(t, \hat{y}^{\rho}(t), \hat{y}^{\nabla}(t)\right) \nabla t$ for $i=k+1, \ldots, k+n$. In contrast with [15], where integral conditions are investigated, here we are interested in obtaining Euler-Lagrange type optimality conditions in differential form.

Remark 3.1. If one considers the particular case where function $H$ in problem (5)-(6) does not depend on nabla operators, then one obtains exactly the delta problem studied in 23. In this case, the assumptions we are considering for problem (5)-(6) coincide with the ones of 23. However, it should be noted that when it is written $\frac{\Delta}{\Delta t}$ or $\frac{\nabla}{\nabla t}$ for some given expression, this is formal and does not mean that one can really expand the delta (or nabla) derivative. Such formal expressions are common in the literature of calculus of variations (see, e.g., [17, Theorem 1 of Section 4], [27, Corollary 2 to Theorem 2.3] or [28, Section 6.1]). All our expressions are valid in integral form (see [15]).

Theorem 3.2 (The delta-nabla Euler-Lagrange equations). Let $\tilde{\mathbb{T}}$ be a time scale with $a, b \in \tilde{\mathbb{T}}$ and $\mathbb{T}:=[a, b] \cap \tilde{\mathbb{T}}$ having at least three points. If $\hat{y}$ is a solution to problem (15)-(6), in the sense of Definition [3.1, then the following delta-nabla Euler-Lagrange equations hold for all $t \in \mathbb{T}_{\kappa}^{\kappa}$ :

$$
\begin{aligned}
& \sum_{i=1}^{k} H_{i}^{\prime} \cdot\left(f_{i y}[\hat{y}](t)-\frac{\Delta}{\Delta t} f_{i v}[\hat{y}](t)\right)+\sum_{i=k+1}^{k+n} H_{i}^{\prime} \cdot\left(f_{i y}\{\hat{y}\}(\sigma(t))-\frac{\Delta}{\Delta t} f_{i v}\{\hat{y}\}(t)\right) \\
&+\frac{\Delta}{\Delta t}\left[\sum_{i=k+1}^{k+n} H_{i}^{\prime} \cdot \nu(t) \cdot\left(f_{i y}\{\hat{y}\}(t)-\frac{\nabla}{\nabla t} f_{i v}\{\hat{y}\}(t)\right)\right]^{\sigma}(t)=0
\end{aligned}
$$

and

$$
\begin{aligned}
\sum_{i=1}^{k} H_{i}^{\prime} \cdot\left(f_{i y}[\hat{y}](\rho(t))-\frac{\nabla}{\nabla t} f_{i v}[\hat{y}](t)\right) & +\sum_{i=k+1}^{k+n} H_{i}^{\prime} \cdot\left(f_{i y}\{\hat{y}\}(t)-\frac{\nabla}{\nabla t} f_{i v}\{\hat{y}\}(t)\right) \\
& -\frac{\nabla}{\nabla t}\left[\sum_{i=1}^{k} H_{i}^{\prime} \cdot \mu(t) \cdot\left(f_{i y}[\hat{y}](t)-\frac{\Delta}{\Delta t} f_{i v}[\hat{y}](t)\right)\right]^{\rho}(t)=0,
\end{aligned}
$$

where $[\hat{y}](t)=\left(t, \hat{y}^{\sigma}(t), \hat{y}^{\Delta}(t)\right)$ and $\{\hat{y}\}(t)=\left(t, \hat{y}^{\rho}(t), \hat{y}^{\nabla}(t)\right)$.

Proof. Suppose that $\mathcal{L}[y]$ has a local extremum at $\hat{y}$. Consider a variation $h \in \mathcal{C}^{1}$ of $\hat{y}$ for which we define the function $\phi: \mathbb{R} \rightarrow \mathbb{R}$ by $\phi(\varepsilon)=\mathcal{L}[\hat{y}+\varepsilon h]$. A necessary condition for $\hat{y}$ to be an extremizer for $\mathcal{L}[y]$ is given by $\phi^{\prime}(\varepsilon)=0$ for $\varepsilon=0$. Using the chain rule, we obtain that

$$
\begin{array}{r}
\phi^{\prime}(0)=\sum_{i=1}^{k} H_{i}^{\prime} \int_{a}^{b}\left(f_{i y}[\hat{y}](t) h^{\sigma}(t)+f_{i v}[\hat{y}](t) h^{\Delta}(t)\right) \Delta t \\
+\sum_{i=k+1}^{k+n} H_{i}^{\prime} \int_{a}^{b}\left(f_{i y}\{\hat{y}\}(t) h^{\rho}(t)+f_{i v}\{\hat{y}\}(t) h^{\nabla}(t)\right) \nabla t=0 .
\end{array}
$$


Using relations

$$
(f g)^{\nabla}(t)=f^{\nabla}(t) g(t)+f^{\rho} g^{\nabla}(t)=f(t) g^{\nabla}(t)+f^{\nabla}(t) g^{\rho}(t)
$$

and

$$
(f g)^{\Delta}(t)=f^{\Delta}(t) g(t)+f^{\sigma} g^{\Delta}(t)=f(t) g^{\Delta}(t)+f^{\Delta}(t) g^{\sigma}(t)
$$

one has

$$
\left(f_{i v}[\hat{y}](t) h(t)\right)^{\Delta}=f_{i v}[\hat{y}](t) h^{\Delta}(t)+\left(f_{i v}[\hat{y}](t)\right)^{\Delta} h^{\sigma}(t)
$$

and

$$
\left(f_{i v}\{\hat{y}\}(t) h(t)\right)^{\nabla}=f_{i v}\{\hat{y}\}(t) h^{\nabla}(t)+\left(f_{i v}\{\hat{y}\}(t)\right)^{\nabla} h^{\rho}(t) .
$$

Integrating both sides from $t=a$ to $t=b$ and having in mind that from (6) one has $h(a)=h(b)=$ 0 , we obtain that

$$
\begin{aligned}
\int_{a}^{b} \sum_{i=1}^{k} H_{i}^{\prime} \cdot\left(f_{i y}[\hat{y}](t)-\left(f_{i v}[\hat{y}](t)\right)^{\Delta}\right) & h^{\sigma}(t) \Delta t \\
& +\int_{a}^{b} \sum_{i=k+1}^{k+n} H_{i}^{\prime} \cdot\left(f_{i y}\{\hat{y}\}(t)-\left(f_{i v}\{\hat{y}\}(t)\right)^{\nabla}\right) h^{\rho}(t) \nabla t=0 .
\end{aligned}
$$

Let us denote

$$
\begin{aligned}
& s(t):=\sum_{i=1}^{k} H_{i}^{\prime} \cdot\left(f_{i y}[\hat{y}](t)-\left(f_{i v}[\hat{y}](t)\right)^{\Delta}\right), \\
& r(t):=\sum_{i=k+1}^{k+n} H_{i}^{\prime} \cdot\left(f_{i y}\{\hat{y}\}(t)-\left(f_{i v}\{\hat{y}\}(t)\right)^{\nabla}\right) .
\end{aligned}
$$

Then,

$$
\int_{a}^{b} s(t) h^{\sigma}(t) \Delta t+\int_{a}^{b} r(t) h^{\rho}(t) \nabla t=0 .
$$

Now we split the proof into two cases. First we use (4) of Theorem 2.14 and (2) of Theorem 2.13 to obtain the Euler-Lagrange equation (7). Next we apply (3) of Theorem 2.14 and (11) of Theorem 2.13 to receive the latter Euler-Lagrange equation (8).

(i) Since $h$ is nabla differentiable, we have that $h^{\rho}(t)=h(t)-\nu(t) h^{\nabla}(t)$ (cf. item (iv) of [2, Theorem 3.2]) and thus

$$
\int_{a}^{b} s(t) h^{\sigma}(t) \Delta t+\int_{a}^{b}\left(r(t) h(t)-r(t) \nu(t) h^{\nabla}(t)\right) \nabla t=0 .
$$

Using equation (4) of Theorem 2.14, it follows that

$$
\int_{a}^{b} s(t) h^{\sigma}(t) \Delta t+\int_{a}^{b}\left[(r h)^{\sigma}(t)-(r \nu)^{\sigma}(t)\left(h^{\nabla}\right)^{\sigma}(t)\right] \Delta t=0 .
$$

Therefore, from equation (2) of Theorem 2.13, we obtain

$$
\int_{a}^{b} s(t) h^{\sigma}(t) \Delta t+\int_{a}^{b}\left[(r h)^{\sigma}(t)-(r \nu)^{\sigma}(t) h^{\Delta}(t)\right] \Delta t=0 .
$$


Integrating the second part of the latter integral gives

$$
\int_{a}^{b}(r \nu)^{\sigma}(t) h^{\Delta}(t) \Delta t=\left.(r \nu)^{\sigma}(t) h(t)\right|_{a} ^{b}-\int_{a}^{b} h^{\sigma}(t) \frac{\Delta}{\Delta t}(r \nu)^{\sigma}(t) \Delta t
$$

and it follows that

$$
\int_{a}^{b}\left[s(t) h^{\sigma}(t)+r^{\sigma}(t) h^{\sigma}(t)+h^{\sigma}(t) \frac{\Delta}{\Delta t}(r \nu)^{\sigma}(t)\right] \Delta t=0 .
$$

Thus,

$$
\int_{a}^{b}\left[s(t)+r^{\sigma}(t)+\frac{\Delta}{\Delta t}(r \nu)^{\sigma}(t)\right] h^{\sigma}(t) \Delta t=0 .
$$

From the fundamental lemma of the delta calculus of variations (cf. Lemma 8 of [1] and Lemma 3.2 of [16]), we get the Euler-Lagrange equation

$$
s(t)+r^{\sigma}(t)+\frac{\Delta}{\Delta t}(r \nu)^{\sigma}(t)=0
$$

and therefore (7) holds.

(ii) Since $h$ is delta differentiable, the following relation holds (cf. item (iv) of [7, Theorem 1.3]):

$$
h^{\sigma}(t)=h(t)+\mu(t) h^{\Delta}(t) .
$$

We then obtain that

$$
\int_{a}^{b} s(t) h(t)+s(t) \mu(t) h^{\Delta}(t) \Delta t+\int_{a}^{b} r(t) h^{\rho}(t) \nabla t=0 .
$$

Using equation (3) of Theorem 2.14,

$$
\int_{a}^{b}\left[s^{\rho}(t) h^{\rho}(t)+(s \mu)^{\rho}(t)\left(h^{\Delta}\right)^{\rho}(t)+r(t) h^{\rho}(t)\right] \nabla t=0 .
$$

It follows, from equation (11) of Theorem 2.13, that

$$
\int_{a}^{b}\left[s^{\rho}(t) h^{\rho}(t)+(s \mu)^{\rho}(t) h^{\nabla}(t)+r(t) h^{\rho}(t)\right] \nabla t=0 .
$$

Integrating the second item of the above integral,

$$
\int_{a}^{b}(s \mu)^{\rho}(t) h^{\nabla}(t) \nabla t=\left.(s \mu)^{\rho}(t) h(t)\right|_{a} ^{b}-\int_{a}^{b} \frac{\nabla}{\nabla t}(s \mu)^{\rho}(t) h^{\rho}(t) \nabla t,
$$

we obtain

$$
\int_{a}^{b}\left[s^{\rho}(t) h^{\rho}(t)+r(t) h^{\rho}(t)-h^{\rho}(t) \frac{\nabla}{\nabla t}(s \mu)^{\rho}(t)\right] \nabla t=0
$$

and then

$$
\int_{a}^{b}\left[s^{\rho}(t)+r(t)-\frac{\nabla}{\nabla t}(s \mu)^{\rho}(t)\right] h^{\rho}(t) \nabla t=0 .
$$


From the fundamental lemma of the nabla calculus of variations (cf. Lemma 15 of [25]), we get the Euler-Lagrange equation

$$
s^{\rho}(t)+r(t)-\frac{\nabla}{\nabla t}(s \mu)^{\rho}(t)=0
$$

and therefore (8) holds.

Corollary 3.1 (Euler-Lagrange equation (3.17) of 11). Let $a, b \in \mathbb{R}$ with $a<b$. If $y$ is solution to problem

$$
\begin{gathered}
\mathcal{L}[y]=H\left(\int_{a}^{b} f_{1}\left(t, y(t), y^{\prime}(t)\right) d t, \int_{a}^{b} f_{2}\left(t, y(t), y^{\prime}(t)\right) d t\right) \longrightarrow \text { extr } \\
y(a)=y_{a}, \quad y(b)=y_{b},
\end{gathered}
$$

then the following differential equation holds:

$$
\begin{aligned}
H_{1}^{\prime}\left(F_{1}, F_{2}\right) \cdot\left(\frac{\partial f_{1}}{\partial y}\left(t, y(t), y^{\prime}(t)\right)\right. & \left.-\frac{d}{d t} \frac{\partial f_{1}}{\partial y^{\prime}}\left(t, y(t), y^{\prime}(t)\right)\right) \\
& +H_{2}^{\prime}\left(F_{1}, F_{2}\right) \cdot\left(\frac{\partial f_{2}}{\partial y}\left(t, y(t), y^{\prime}(t)\right)-\frac{d}{d t} \frac{\partial f_{2}}{\partial y^{\prime}}\left(t, y(t), y^{\prime}(t)\right)\right)=0
\end{aligned}
$$

for all $t \in[a, b]$, where

$$
F_{i}=\int_{a}^{b} f_{i}\left(t, y(t), y^{\prime}(t)\right) d t, \quad i=1,2 .
$$

Proof. Let $\tilde{\mathbb{T}}=\mathbb{R}$ and $k=n=1$. The result follows from Theorem 3.2

Let $\varphi: \mathbb{R}^{3} \rightarrow \mathbb{R}$. In what follows we use $\partial_{i} \varphi, i \in\{1,2,3\}$, to denote the partial derivative of $\varphi$ with respect to its $i$ th argument.

Corollary 3.2. Let $a, b \in \mathbb{N}$ with $b-a>1$ and denote by $\Delta y(t)$ and $\nabla y(t)$ the standard forward and backward difference operators, that is, $\Delta y(t):=y(t+1)-y(t)$ and $\nabla y(t):=y(t)-y(t-1)$. If $y$ is solution to problem

$$
\begin{gathered}
\mathcal{L}[y]=H\left(\sum_{t=a}^{b-1} f(t, y(t+1), \Delta y(t)), \sum_{t=a+1}^{b} g(t, y(t-1), \nabla y(t))\right) \longrightarrow \text { extr } \\
y(a)=y_{a}, \quad y(b)=y_{b},
\end{gathered}
$$

then both Euler-Lagrange difference equations

$$
\begin{aligned}
H_{1}^{\prime}(F, G) \cdot[ & \left.\partial_{2} f(t, y(t+1), \Delta y(t))-\Delta \partial_{3} f(t, y(t+1), \Delta y(t))\right] \\
& +H_{2}^{\prime}(F, G) \cdot\left[\partial_{2} g(t+1, y(t), \nabla y(t+1))-\Delta \partial_{3} g(t, y(t-1), \nabla y(t))\right] \\
& +H_{2}^{\prime}(F, G) \cdot \Delta\left[\partial_{2} g(t+1, y(t), \nabla y(t+1))-\nabla \partial_{3} g(t+1, y(t), \nabla y(t+1))\right]=0
\end{aligned}
$$

and

$$
\begin{aligned}
H_{1}^{\prime}(F, G) \cdot\left[\partial_{2} f(t-1, y(t), \Delta y(t-1))-\nabla \partial_{3} f(t, y(t+1), \Delta y(t))\right] & \\
-H_{1}^{\prime}(F, G) & \cdot \nabla\left[\partial_{2} f(t-1, y(t), \Delta y(t-1))-\Delta \partial_{3} f(t-1, y(t), \Delta y(t-1))\right] \\
& +H_{2}^{\prime}(F, G) \cdot\left[\partial_{2} g(t, y(t-1), \nabla y(t))-\nabla \partial_{3} g(t, y(t-1), \nabla y(t))\right]=0
\end{aligned}
$$

hold for $t \in\{a+1, \ldots, b-1\}$, where

$$
F:=\sum_{t=a}^{b-1} f(t, y(t+1), \Delta y(t)), \quad G:=\sum_{t=a+1}^{b} g(t, y(t-1), \nabla y(t)) .
$$

Proof. The result is a direct consequence of Theorem 3.2 with $\tilde{\mathbb{T}}=\mathbb{Z}$ and $k=n=1$. 


\section{Application to Economics}

In this section we introduce an economic problem that is considered in continuous (Example 4.1) and discrete (Example 4.2) cases. We consider a firm that wants to program its production and investment policies in order to gain a desirable production level and maximize its market competitiveness. Our idea is to discretize necessary optimality conditions of Euler-Lagrange type $\left(E L_{P}\right)$ and the (continuous) problem $P$ in different ways, combining forward $(\Delta)$ and backward $(\nabla)$ discretization operators into a mixed operator $D$. One can apply the variational principle to problem $P$ obtaining the respective Euler-Lagrange equation $E L_{P}$ (Corollary 3.1), and then discretize it using $D$, obtaining $\left(E L_{P}\right)_{D}$; or we can begin by discretizing problem $P$ into $P_{D}$ and then develop the respective variational principle, obtaining $E L_{P_{D}}$ (Theorem 3.2). This is illustrated in Figure 1. Note that, in general, $\left(E L_{P}\right)_{D}$ is different from $E L_{P_{D}}$. Four different

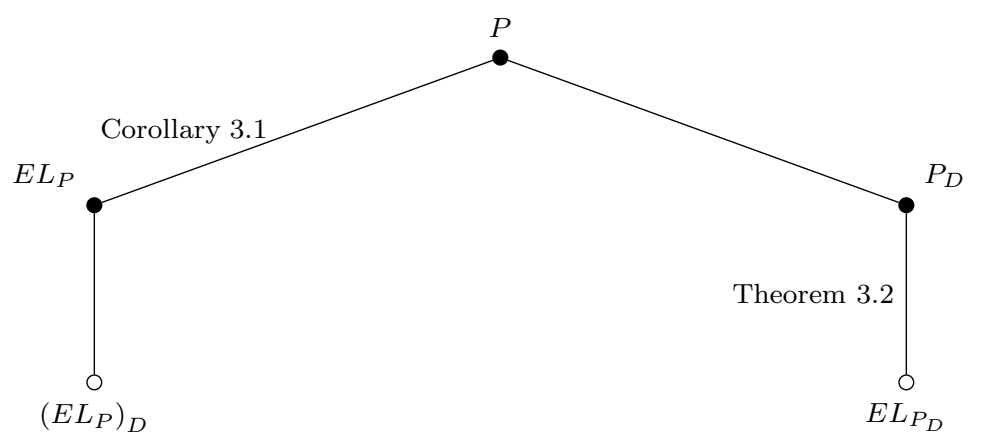

Figure 1: Diagram illustrating different discretizations for a variational problem $P$.

problems $P_{D}$, four Euler-Lagrange equations $E L_{P_{D}}$ and four Euler-Lagrange equations $\left(E L_{P}\right)_{D}$ are discussed and investigated. In what follows,

$$
\Delta y(t):=y^{\sigma}(t)-y(t), \quad \nabla y(t):=y(t)-y^{\rho}(t) .
$$

In particular, if $\mathbb{T}$ has a maximum $M$, then $\Delta y(M)=0$; if $\mathbb{T}$ has a minimum $m$, then $\nabla y(m)=0$.

\subsection{Direct discretizations of the continuous Euler-Lagrange equation}

The next example is borrowed from Section 6 of [11].

Example 4.1 (A continuous problem of the calculus of variations [11]). We consider a firm trying to program its production and investment policies in order to reach a desirable production level and to maximize its future market competitiveness at time horizon $T$. The firm competitiveness is measured by the function $f(k(T), a(T)$ ), which depends on the accumulated capital $k(T)$ (accumulated goods devoted to production) and accumulated technology $a(T)$ (capability given by the practical application of knowledge and experience), both at time horizon $T$. We assume that the function measuring the firm market competitiveness is the product of the accumulated capital with the accumulated technology, that is,

$$
f(k(T), a(T))=k(T)^{\gamma_{1}} a(T)^{\gamma_{2}},
$$

where $\gamma_{1}$ and $\gamma_{2}$ are constants measuring the absolute and relative importance/influence of capital and technology competitiveness, respectively. More precisely, the firm may decide to sell products at a small or no benefit, or even losses, if due to this decision the firm can gain experience and get technology acquisition. Firm's measure of competitiveness is chosen as the product of two components because there is a strict dependence between capital and technology. Indeed, one affects the other, and huge differences between them cannot be allowed. This means that a lack 
of one of those components must be compensated by a large amount of the other in order to reach the same competitiveness level. The firm starts operating at time $t=0$ and accumulates capital over time as

$$
k(T)=\int_{0}^{T} e^{-\rho(T-t)}\left[y(t) p(t)-c\left(y(t), y^{\prime}(t)\right)\right] d t,
$$

where $\rho$ is the discount rate, $p(t)$ is the unit product price at time $t$, and $y(t)$ is the actual production rate at time $t$. The accumulated capital depends also on function $c\left(y(t), y^{\prime}(t)\right)$, which is the cost of producing $y(t)$ units of product at time $t$ plus technology increases. In our model the cost function has the following form:

$$
c\left(y(t), y^{\prime}(t)\right)=c_{0}+c_{1} y(t)+c_{2} y^{\prime 2}(t),
$$

where $c_{0}, c_{1}, c_{2}$ are positive constants. The second component of (12) is the accumulated technology, which is the discounted integral of the technology acquisition rate over time:

$$
a(T)=\int_{0}^{T} e^{-\rho(T-t)} g\left(y(t), y^{\prime}(t)\right) d t
$$

with

$$
g\left(y(t), y^{\prime}(t)\right)=\lambda y(t)+\beta \sqrt{y^{\prime}(t)+b},
$$

where $\lambda, \beta$ and $b$ are positive constants. Function $g$ describes the acquisition technology rate at time $t$. It depends on the actual sales rate $y(t)$ (equal to the actual production rate at the same time) and $y^{\prime}(t)$, the actual production rate change. The $y(t)$ argument accounts for machines, other technology components, gained experience, etc., while the $y^{\prime}(t)$ argument accounts for technology due to changes on sales rate. This means that large positive or negative changes on sales rate $y^{\prime}(t)$ forces the firm to make decisions about technology increases: it may be a starting point for the increase of production or a warning to avoid decrease. All constants used in the model are positive and have a precise interpretation. It is also worth to emphasise that both the production cost function $c$ and the acquisition technology function $g$ depend on argument $y^{\prime}$. However, in the first function, argument $y^{\prime}$ is of higher-order than in the latter, motivated by the fact that incorporation of technology into a production process is very difficult, generates costs, and requires time to be checked. The sales relationship is given by

$$
h(y(t), p(t))=\left(y(t)-y_{0}\right)\left(p(t)-p_{0}\right)-B=0 .
$$

It has this hyperbolic form in order to express the assumption that sales increase when the unit price decrease. Moreover, it also gives the lower limit for the sales $\left(y_{0}\right)$ and the lower limit for the unit price $\left(p_{0}\right)$. There is an upper bound $b$ for the size of production rate change so that $\left|y^{\prime}\right| \leq b$. The economic problem under consideration is

$$
\max _{y} f(k(T), a(T))=\min _{y}[-k(T) a(T)]=\min _{y} K(T) a(T),
$$

where $K(T)=-k(T)$. More precisely, we consider $\gamma_{1}=\gamma_{2}=1$, and the problem takes the form

$$
\min _{y}\left(\int_{0}^{T} e^{-\rho(T-t)}\left[c\left(y(t), y^{\prime}(t)\right)-y(t) p(t)\right] d t\right)\left(\int_{0}^{T} e^{-\rho(T-t)} g\left(y(t), y^{\prime}(t)\right) d t\right)
$$

subject to given boundary conditions

$$
y(0)=y_{0}, \quad y(T)=y_{T},
$$

where $y_{0}$ is the initial sales rate and $y_{T}$ is the target sales rate at time $t=T$. This problem is denoted in the sequel by $(P)$. Note that here $\rho$ is the discount rate (not to be confused with the 
backward jump operator $\rho(t)$ of time scales, which in the discrete case, to be considered in this section, is always expressed by the index $k-1)$. For problem $(P)$ the Euler-Lagrange differential equation (9) takes the form

$$
\begin{aligned}
a(T) e^{-\rho(T-t)}\left[c_{1}-p(t)-\right. & \left.2 c_{2}\left(\rho y^{\prime}(t)+y^{\prime \prime}(t)\right)\right] \\
& +K(T) e^{-\rho(T-t)}\left[\lambda-\frac{\beta}{2}\left(\frac{\rho}{\sqrt{y^{\prime}(t)+b}}-\frac{y^{\prime \prime}(t)}{2 \sqrt{\left(y^{\prime}(t)+b\right)^{3}}}\right)\right]=0 .
\end{aligned}
$$

The solution of the continuous problem $(P)$ is found by solving the Euler-Lagrange equation (13). It turns out that this is a highly nonlinear differential equation of second order, for which no analytical solution is known. In other words, to solve the continuous problem one needs to apply a suitable discretization. This is exactly one of the main motivations of our study: to provide an appropriate theory of discretization.

A discretization can always be done in two different ways: using the delta or the nabla approach. In the next example we consider four different discretizations for the problem $(P)$ of Example 4.1 and the corresponding four discretizations of the Euler-Lagrange equation (13).

Example 4.2. Consider a firm that wants to program its production and investment policies to reach a given production rate $k(T), T \in \mathbb{N}$, and to maximize its future market competitiveness at time horizon $T$. Economic models, leading to the maximization of a variational functional, are presented below and are based on the following assumptions:

1. The firm competitiveness is measured by the function $f(k(T), a(T))$, which depends on the accumulated capital $k(T)$ and on the accumulated technology $a(T)$ both at time horizon $T$. Here, the function to measure the firm market competitiveness is assumed to be of form

$$
f(k(T), a(T))=k(T)^{\gamma_{1}} a(T)^{\gamma_{2}}
$$

with $\gamma_{1}$ and $\gamma_{2}$ given constants that measure the absolute and relative importance of capital and technology competitiveness, respectively.

2. The acquisition technology rate is given by function $g\left(y\left(t_{k+1}\right), \Delta y\left(t_{k}\right)\right)$ (delta version) or $g\left(y\left(t_{k-1}\right), \nabla y\left(t_{k}\right)\right)$ (nabla version), where $y(s)$ is the sales rate at time $s$, which we assume equal to the actual production rate at the same point of time, that is, $\Delta y\left(t_{k}\right)$ (delta version) or $\nabla y\left(t_{k}\right)$ (nabla version) are the actual production rate change at time $t_{k}$.

3. The firm starts operating at point $t_{0}=0$ and accumulates capital as

$$
K_{\Delta}(T)=\sum_{t_{k}=0}^{T-1}(1+\rho)^{t_{k}-T}\left(c_{0}+c_{1} y_{k+1}+c_{2}\left(\Delta y_{k}\right)^{2}-y_{k+1} p_{k+1}\right)
$$

(delta version) or

$$
K_{\nabla}(T)=\sum_{t_{k}=1}^{T}(1-\rho)^{T-t_{k}}\left(c_{0}+c_{1} y_{k-1}+c_{2}\left(\nabla y_{k}\right)^{2}-y_{k-1} p_{k-1}\right)
$$

(nabla version), where $\rho$ is the discount rate, $p_{k}=p\left(t_{k}\right)$ is the unit product price, $y_{k}=y\left(t_{k}\right)$ is the sales rate at time $t_{k}$, and $c\left(y_{k+1}, \Delta y_{k}\right)$ (delta) or $c\left(y_{k-1}, \nabla y_{k}\right)$ (nabla) is the cost of producing $y_{k+1}$ (delta) or $y_{k-1}$ (nabla) units of product at time $t_{k+1}$ (delta) or $t_{k-1}$ (nabla) plus technology increases.

4. The accumulate technology is given by

$$
a_{\Delta}(T)=\sum_{t_{k}=0}^{T-1}(1+\rho)^{t_{k}-T}\left(\lambda y_{k+1}+\beta \sqrt{\Delta y_{k}+b}\right)
$$


(delta version) or

$$
a_{\nabla}(T)=\sum_{t_{k}=1}^{T}(1-\rho)^{T-t_{k}}\left(\lambda y_{k-1}+\beta \sqrt{\nabla y_{k}+b}\right)
$$

(nabla version).

5. The price-sales relationship regulating the market is given by the equation

$$
h\left(y_{k+1}, p_{k+1}\right)=\left(y_{k+1}-y_{0}\right)\left(p_{k+1}-p_{0}\right)-B=0
$$

(delta version) or by the equation

$$
h\left(y_{k-1}, p_{k-1}\right)=\left(y_{k-1}-y_{0}\right)\left(p_{k-1}-p_{0}\right)-B=0
$$

(nabla version). There is an upper bound $b$ for the size of production rate change, so that $\left|\Delta y_{k}\right| \leq b$ (delta) or $\left|\nabla y_{k}\right| \leq b$ (nabla).

6. Two boundary conditions are given:

$$
y(0)=y_{0}, \quad y(T)=y_{T},
$$

which are the initial sales rate at point $t_{0}=0$ and the target sales rate at the terminal point of time $t_{k}=T$.

Then, the firm problem is stated as follows:

$$
\max _{y_{k}} k(T)^{\gamma_{1}} a(T)^{\gamma_{2}}
$$

subject to the hypotheses (14)-(21). For illustrative purposes, and to be coherent with Example4.1 borrowed from [11], we assume $\gamma_{1}=\gamma_{2}=1$ and we transform the maximization problem into an equivalent minimization process:

$$
\min _{y_{k}}(-k(T)) a(T)=\min _{y_{k}} K(T) a(T) .
$$

Each component of the objective functional $f(K(T), a(T))$ may be discretized in two ways (using the delta or the nabla approach). Due to this reason, we obtain four different discrete problems of the calculus of variations:

1. Problem $\left(P_{\Delta \nabla}\right)$ with cost functional $\min _{y_{k}} K_{\Delta}(T) a_{\nabla}(T)$;

2. Problem $\left(P_{\nabla \Delta}\right)$ with cost functional $\min K_{\nabla}(T) a_{\Delta}(T)$;

3. Problem $\left(P_{\Delta \Delta}\right)$ with cost functional $\min _{y_{k}} K_{\Delta}(T) a_{\Delta}(T)$;

4. Problem $\left(P_{\nabla \nabla}\right)$ with cost functional $\min _{y_{k}} K_{\nabla}(T) a_{\nabla}(T)$;

where $K_{\mathcal{D}}(T)$ and $a_{\mathcal{D}}(T), \mathcal{D} \in\{\Delta, \nabla\}$, are defined as in (15)-(18). With the notation of Section 3 , such functionals consist of the following integrands:

$$
\begin{aligned}
& f_{1 \Delta}=(1+\rho)^{t_{k}-T}\left(c_{0}+c_{1} y_{k+1}+c_{2}\left(\Delta y_{k}\right)^{2}-y_{k+1} p_{k+1}\right), \\
& f_{1 \nabla}=(1-\rho)^{T-t_{k}}\left(c_{0}+c_{1} y_{k-1}+c_{2}\left(\nabla y_{k}\right)^{2}-y_{k-1} p_{k-1}\right), \\
& f_{2 \Delta}=(1+\rho)^{t_{k}-T}\left(\lambda y_{k+1}+\beta \sqrt{\Delta y_{k}+b}\right), \\
& f_{2 \nabla}=(1-\rho)^{T-t_{k}}\left(\lambda y_{k-1}+\beta \sqrt{\nabla y_{k}+b}\right),
\end{aligned}
$$


where $f_{i \Delta}=f_{i \Delta}\left(t_{k}, y_{k+1}, \Delta y_{k}\right), f_{i \nabla}=f_{i \nabla}\left(t_{k}, y_{k-1}, \nabla y_{k}\right), i=1,2$, and function $f_{1 \mathcal{D}}$ is associated with functional $K_{\mathcal{D}}(T)$ and function $f_{2 \mathcal{D}}$ is associated with functional $a_{\mathcal{D}}(T), \mathcal{D} \in\{\Delta, \nabla\}$. Using the same discretization as the one from $(P)$ to $\left(P_{\Delta \nabla}\right)$, the Euler-Lagrange equation (9) is discretized into

$$
a_{\nabla}(T) \cdot\left(\frac{\partial f_{1 \Delta}}{\partial y_{k+1}}-\Delta \frac{\partial f_{1 \Delta}}{\partial \Delta y_{k}}\right)+K_{\Delta}(T) \cdot\left(\frac{\partial f_{2 \nabla}}{\partial y_{k-1}}-\nabla \frac{\partial f_{2 \nabla}}{\partial \nabla y_{k}}\right)=0,
$$

which for our economic problem $(P)$ takes the form

$$
\begin{aligned}
a_{\nabla}(T)(1+\rho)^{t_{k}-T} & {\left[c_{1}-p_{0}+\frac{B y_{0}}{\left(y_{k+1}-y_{0}\right)^{2}}-2 c_{2}\left(\rho \Delta y_{k}+(1+\rho) \Delta^{2} y_{k}\right)\right] } \\
& +K_{\Delta}(T)(1-\rho)^{T-t_{k}}\left[\lambda-\frac{\beta\left(\rho \sqrt{\nabla y_{k}+b}-\nabla \sqrt{\nabla y_{k}+b}\right)}{2 \sqrt{\nabla y_{k}+b} \sqrt{\nabla y_{k-1}+b}}\right]=0, \quad\left(\left(E L_{P}\right)_{\Delta \nabla}\right)
\end{aligned}
$$

valid for $t_{k} \in \mathbb{T}_{\kappa}^{\kappa}$. Note that we start with a given value of sales (or production) rate $y_{0}$ that the firm wants to improve (increase) in order to generate a profit. For this reason, the next values $y_{k}$, $k>0$, are assumed to be greater than the initial value $y_{0}$. This economic assumption, makes valid the Euler-Lagrange equation $\left.\left(E L_{P}\right)_{\Delta \nabla}\right)$. Indeed, it is known a priori, from economic insight, that $y(t)$ is an increasing function [11]. Similarly, the discretization from $(P)$ into $\left(P_{\nabla \Delta}\right)$ gives the discretized Euler-Lagrange equation

$$
a_{\Delta}(T) \cdot\left(\frac{\partial f_{1 \nabla}}{\partial y_{k-1}}-\nabla \frac{\partial f_{1 \nabla}}{\partial \nabla y_{k}}\right)+K_{\nabla}(T) \cdot\left(\frac{\partial f_{2 \Delta}}{\partial y_{k+1}}-\Delta \frac{\partial f_{2 \Delta}}{\partial \Delta y_{k}}\right)=0
$$

that, for our example, reads

$$
\begin{aligned}
a_{\Delta}(T)(1-\rho)^{T-t_{k}} & {\left[c_{1}-p_{0}+\frac{B y_{0}}{\left(y_{k-1}-y_{0}\right)^{2}}-2 c_{2}\left(\rho \nabla y_{k}+(1-\rho) \nabla^{2} y_{k}\right)\right] } \\
& +K_{\nabla}(T)(1+\rho)^{t_{k}-T}\left[\lambda-\frac{\beta\left(\rho \sqrt{\Delta y_{k}+b}-\Delta \sqrt{\Delta y_{k}+b}\right)}{2 \sqrt{\Delta y_{k}+b} \sqrt{\Delta y_{k+1}+b}}\right]=0, \quad\left(\left(E L_{P}\right)_{\nabla \Delta}\right)
\end{aligned}
$$

$t_{k} \in \mathbb{T}_{\kappa}^{\kappa}$; the discretization from $(P)$ into $\left(P_{\Delta \Delta}\right)$ leads to the discretized Euler-Lagrange equation

$$
a_{\Delta}(T) \cdot\left(\frac{\partial f_{1 \Delta}}{\partial y_{k+1}}-\Delta \frac{\partial f_{1 \Delta}}{\partial \Delta y_{k}}\right)+K_{\Delta}(T) \cdot\left(\frac{\partial f_{2 \Delta}}{\partial y_{k+1}}-\Delta \frac{\partial f_{2 \Delta}}{\partial \Delta y_{k}}\right)=0
$$

and to

$$
\begin{aligned}
a_{\Delta}(T)(1+\rho)^{t_{k}-T} & {\left[c_{1}-p_{0}+\frac{B y_{0}}{\left(y_{k+1}-y_{0}\right)^{2}}-2 c_{2}\left(\rho \Delta y_{k}+(1+\rho) \Delta^{2} y_{k}\right)\right] } \\
& +K_{\Delta}(T)(1+\rho)^{t_{k}-T}\left[\lambda-\frac{\beta\left(\rho \sqrt{\Delta y_{k}+b}-\Delta \sqrt{\Delta y_{k}+b}\right)}{2 \sqrt{\Delta y_{k}+b} \sqrt{\Delta y_{k+1}+b}}\right]=0, \quad\left(\left(E L_{P}\right)_{\Delta \Delta}\right)
\end{aligned}
$$

$t_{k} \in \mathbb{T}^{\kappa^{2}}$; while the discretization from $(P)$ into problem $\left(P_{\nabla \nabla}\right)$ gives

$$
a_{\nabla}(T) \cdot\left(\frac{\partial f_{1 \nabla}}{\partial y_{k-1}}-\nabla \frac{\partial f_{1 \nabla}}{\partial \nabla y_{k}}\right)+K_{\nabla}(T) \cdot\left(\frac{\partial f_{2 \nabla}}{\partial y_{k-1}}-\nabla \frac{\partial f_{2 \nabla}}{\partial \nabla y_{k}}\right)=0
$$

that reduces in our case to

$$
\begin{aligned}
a_{\nabla}(T)(1-\rho)^{T-t_{k}} & {\left[c_{1}-p_{0}+\frac{B y_{0}}{\left(y_{k-1}-y_{0}\right)^{2}}-2 c_{2}\left(\rho \nabla y_{k}+(1-\rho) \nabla^{2} y_{k}\right)\right] } \\
& +K_{\nabla}(T)(1-\rho)^{T-t_{k}}\left[\lambda-\frac{\beta\left(\rho \sqrt{\nabla y_{k}+b}-\nabla \sqrt{\nabla y_{k}+b}\right)}{2 \sqrt{\nabla y_{k}+b} \sqrt{\nabla y_{k-1}+b}}\right]=0, \quad\left(\left(E L_{P}\right)_{\nabla \nabla}\right)
\end{aligned}
$$


valid for $t_{k} \in \mathbb{T}_{\kappa^{2}}$. As can be easily noticed, all the four discretizations of the continuous EulerLagrange equation (9) are different but consist of the same items. For this reason, we define:

$$
\begin{array}{ll}
\gamma_{1 \Delta}:=\left(\frac{\partial f_{1 \Delta}}{\partial y_{k+1}}-\Delta \frac{\partial f_{1 \Delta}}{\partial \Delta y_{k}}\right), \quad \gamma_{1 \nabla}:=\left(\frac{\partial f_{1 \nabla}}{\partial y_{k-1}}-\nabla \frac{\partial f_{1 \nabla}}{\partial \nabla y_{k}}\right), \\
\gamma_{2 \Delta}:=\left(\frac{\partial f_{2 \Delta}}{\partial y_{k+1}}-\Delta \frac{\partial f_{2 \Delta}}{\partial \Delta y_{k}}\right), \quad \gamma_{2 \nabla}:=\left(\frac{\partial f_{2 \nabla}}{\partial y_{k-1}}-\nabla \frac{\partial f_{2 \nabla}}{\partial \nabla y_{k}}\right) .
\end{array}
$$

With such notations, the discretizations of the Euler-Lagrange equation (9) are conveniently written in the following way:

1. equation (22) for $\left(P_{\Delta \nabla}\right)$ is written as

$$
a_{\nabla}(T) \gamma_{1 \Delta}+K_{\Delta}(T) \gamma_{2 \nabla}=0, \quad t_{k} \in \mathbb{T}_{\kappa}^{\kappa} ;
$$

2. equation (23) for $\left(P_{\nabla \Delta}\right)$ is written as

$$
a_{\Delta}(T) \gamma_{1 \nabla}+K_{\nabla}(T) \gamma_{2 \Delta}=0, \quad t_{k} \in \mathbb{T}_{\kappa}^{\kappa}
$$

3. equation (24) for $\left(P_{\Delta \Delta}\right)$ is written as

$$
a_{\Delta}(T) \gamma_{1 \Delta}+K_{\Delta}(T) \gamma_{2 \Delta}=0, \quad t_{k} \in \mathbb{T}^{\kappa^{2}}
$$

4. and equation (25) for $\left(P_{\nabla \nabla}\right)$ is equivalently written as

$$
a_{\nabla}(T) \gamma_{1 \nabla}+K_{\nabla}(T) \gamma_{2 \nabla}=0, \quad t_{k} \in \mathbb{T}_{\kappa^{2}} .
$$

\subsection{Time-scale Euler-Lagrange equations}

The equation (28) for problem $\left(P_{\Delta \Delta}\right)$ coincides with the time-scale Euler-Lagrange delta equation given by [23, Corollary 3.4] while equation (29) for problem $\left(P_{\nabla \nabla}\right)$ coincides with the time-scale Euler-Lagrange equation given by [24, Corollary 3.4]. From our Corollary 3.2 it follows that such coincidence, between the direct discretization of the continuous Euler-Lagrange equation (9) and the discrete Euler-Lagrange equations (10)-(11) obtained from the calculus of variations on time scales, does not hold for mixed delta-nabla discretizations: neither (26) is a time-scale EulerLagrange equation (10) or (11) for $\left(P_{\Delta \nabla}\right)$ nor (27) is a time-scale Euler-Lagrange equation (10) or (11) for $\left(P_{\nabla \Delta}\right)$.

For the economic problem $\left(P_{\Delta \nabla}\right)$ the Euler-Lagrange equations have the following form: the Euler-Lagrange equation (10) takes the form

$$
\begin{aligned}
& a_{\nabla}(T)(1+\rho)^{t_{k}-T}\left[c_{1}-p_{0}+\frac{B y_{0}}{\left(y_{k+1}-y_{0}\right)^{2}}-2 c_{2}\left(\rho \Delta y_{k}+(1+\rho) \Delta^{2} y_{k}\right)\right] \\
& +K_{\Delta}(T)(1-\rho)^{T-t_{k}}\left(\lambda-\frac{\beta\left(\rho \sqrt{\nabla y_{k}+b}-(1-\rho) \Delta \sqrt{\nabla y_{k}+b}\right)}{2 \sqrt{\nabla y_{k}+b} \sqrt{\nabla y_{k+1}+b}}\right) \\
& +\Delta\left[K_{\Delta}(T)(1-\rho)^{T-t_{k}}\left(\lambda-\frac{\beta\left(\rho \sqrt{\nabla y_{k}+b}-\nabla \sqrt{\nabla y_{k}+b}\right)}{2 \sqrt{\nabla y_{k}+b} \sqrt{\nabla y_{k-1}+b}}\right)\right]\left(t_{k+1}\right)=0
\end{aligned}
$$

for $t_{k} \in \mathbb{T}_{\kappa}^{\kappa}$, while the Euler-Lagrange equation (11) gives

$$
\begin{aligned}
& a_{\nabla}(T)(1+\rho)^{t_{k-1}-T}\left[c_{1}-p_{0}+\frac{B y_{0}}{\left(y_{k}-y_{0}\right)^{2}}-2 c_{2}\left(\rho \Delta y_{k}+\nabla\left(\Delta y_{k}\right)\right)\right] \\
& +K_{\Delta}(T)(1-\rho)^{T-t_{k}}\left(\lambda-\frac{\beta\left(\rho \sqrt{\nabla y_{k 1}+b}-\nabla \sqrt{\nabla y_{k}+b}\right)}{2 \sqrt{\nabla y_{k}+b} \sqrt{\nabla y_{k-1}+b}}\right) \\
& -\nabla\left[a_{\nabla}(T)(1+\rho)^{t_{k}-T}\left(c_{1}-p_{0}+\frac{B y_{0}}{\left(y_{k}-y_{0}\right)^{2}}-2 c_{2}\left(\rho \Delta y_{k}+(1+\rho) \Delta^{2} y_{k}\right)\right)\right]\left(t_{k-1}\right)=0
\end{aligned}
$$

$\left(E L_{P_{\Delta \nabla}}^{2}\right)$ 
for $t_{k} \in \mathbb{T}_{\kappa}^{\kappa}$. For problem $\left(P_{\nabla \Delta}\right)$ the Euler-Lagrange equations take the following form: the Euler-Lagrange equation (10) gives

$$
\begin{aligned}
& a_{\Delta}(T)(1-\rho)^{T-t_{k}-1}\left[c_{1}-p_{0}+\frac{B y_{0}}{\left(y_{k}-y_{0}\right)^{2}}-2 c_{2}\left(\rho \nabla y_{k}+\Delta\left(\nabla y_{k}\right)\right)\right] \\
& +K_{\nabla}(T)(1+\rho)^{t_{k}-T}\left[\lambda-\frac{\beta\left(\rho \sqrt{\Delta y_{k}+b}-\Delta \sqrt{\Delta y_{k}+b}\right)}{2 \sqrt{\Delta y_{k}+b} \sqrt{\Delta y_{k+1}+b}}\right] \\
& +\Delta\left[a_{\Delta}(T)(1-\rho)^{T-t_{k}}\left[c_{1}-p_{0}+\frac{B y_{0}}{\left(y_{k-1}-y_{0}\right)^{2}}-2 c_{2}\left(\rho \nabla y_{k}+(1-\rho) \nabla^{2} y_{k}\right)\right]\right]\left(t_{k+1}\right)=0
\end{aligned}
$$

for $t_{k} \in \mathbb{T}_{\kappa}^{\kappa}$, and (11) gives

$$
\begin{array}{ll}
a_{\Delta}(T)(1-\rho)^{T-t_{k}}\left[c_{1}-p_{0}+\frac{B y_{0}}{\left(y_{k-1}-y_{0}\right)^{2}}-2 c_{2}\left(\rho \nabla y_{k}+(1-\rho) \nabla^{2} y_{k}\right)\right] & \\
+K_{\nabla}(T)(1+\rho)^{t_{k-1}-T}\left[\lambda-\frac{\beta\left(\rho \sqrt{\Delta y_{k}+b}-(1+\rho) \nabla \sqrt{\Delta y_{k}+b}\right)}{2 \sqrt{\Delta y_{k}+b} \sqrt{\nabla y_{k}+b}}\right] & \left(E L_{P_{\nabla \Delta}}^{2}\right) \\
-\nabla\left[K_{\nabla}(T)(1+\rho)^{t_{k}-T}\left[\lambda-\frac{\beta\left(\rho \sqrt{\Delta y_{k}+b}-\Delta \sqrt{\Delta y_{k}+b}\right)}{2 \sqrt{\Delta y_{k}+b} \sqrt{\Delta y_{k+1}+b}}\right]\right] & \left(t_{k-1}\right)=0
\end{array}
$$

for $t_{k} \in \mathbb{T}_{\kappa}^{\kappa}$. Then the Euler-Lagrange equations $E L_{P_{\Delta \nabla}}^{1}$ and $E L_{P_{\Delta \nabla}}^{2}$ for $\left(P_{\Delta \nabla}\right)$ are

$$
a_{\nabla}(T) \gamma_{1 \Delta}+K_{\Delta}(T)\left(\frac{\partial f_{2 \nabla}}{\partial y_{k-1}} \circ \sigma-\Delta \frac{\partial f_{2 \nabla}}{\partial \nabla y_{k}}\right)+\Delta\left[K_{\Delta}(T) \gamma_{2 \nabla}\right] \circ \sigma=0, \quad t_{k} \in \mathbb{T}_{\kappa}^{\kappa},
$$

and

$$
a_{\nabla}(T)\left(\frac{\partial f_{1 \Delta}}{\partial y_{k+1}} \circ \rho-\nabla \frac{\partial f_{1 \Delta}}{\partial \Delta y_{k}}\right)+K_{\Delta}(T) \gamma_{2 \nabla}-\nabla\left[a_{\nabla}(T) \gamma_{1 \Delta}\right] \circ \rho=0, \quad t_{k} \in \mathbb{T}_{\kappa}^{\kappa},
$$

respectively, and the Euler-Lagrange equations $E L_{P_{\nabla \Delta}}^{1}$ and $E L_{P_{\nabla \Delta}}^{2}$ for $\left(P_{\nabla \Delta}\right)$ are

$$
a_{\Delta}(T)\left(\frac{\partial f_{1 \nabla}}{\partial y_{k-1}} \circ \sigma-\Delta \frac{\partial f_{1 \nabla}}{\partial \nabla y_{k}}\right)+K_{\nabla}(T) \gamma_{2 \Delta}+\Delta\left[a_{\Delta}(T) \gamma_{1 \nabla}\right] \circ \sigma=0, \quad t_{k} \in \mathbb{T}_{\kappa}^{\kappa},
$$

and

$$
a_{\Delta}(T) \gamma_{1 \nabla}+K_{\nabla}(T)\left(\frac{\partial f_{2 \Delta}}{\partial y_{k+1}} \circ \rho-\nabla \frac{\partial f_{2 \Delta}}{\partial \Delta y_{k}}\right)-\nabla\left[K_{\nabla}(T) \gamma_{2 \Delta}\right] \circ \rho=0, \quad t_{k} \in \mathbb{T}_{\kappa}^{\kappa},
$$

respectively.

For the convenience of the reader, we recall the introduced notations:

- $P$ - the continuous economic problem describing a market policy of a firm, presented in Section 4

- $E L_{P}$ - the continuous Euler-Lagrange equation (9) associated to problem $P$ (see (13));

- $P_{D}$ - a discretization of problem $P$, in four possible forms: $D \in\{\Delta \Delta, \nabla \nabla, \Delta \nabla, \nabla \Delta\}$;

- $\left(E L_{P}\right)_{D}$ - a discretization of the Euler-Lagrange equation $E L_{P}$, in four different forms: $D \in\{\Delta \Delta, \nabla \nabla, \Delta \nabla, \nabla \Delta\}$

- $E L_{P_{D}}$ - discrete Euler-Lagrange equations associated to problem $P_{D}$, obtained from the calculus of variations on time scales (see Corollary 3.2). 


\section{$5 \quad$ Standard versus time-scale discretizations}

The discrepancy between direct discretization of the classical optimality conditions and the timescale approach to the calculus of variations was discussed, from an embedding point of view, in [12. Here we compare the results obtained from direct and time-scale discretizations for the more general problem (5) -(6), in concrete for the economic problem $(P)$ discussed in Section 45 For illustrative purposes, the following values have been borrowed from [11:

$$
\begin{gathered}
\rho=0.05, \quad c_{0}=3, \quad c_{1}=0.5, \quad c_{2}=3, \quad T=3, \\
b=4, \quad \lambda=\frac{1}{2}, \quad \beta=\frac{1}{4}, \quad B=2, \quad y_{0}=2, \quad y_{T}=3 .
\end{gathered}
$$

Moreover, we fixed the time scale to be $\mathbb{T}=\{0,1,2,3\}$. In what follows we compare the candidates for solutions of the variational problems $\left(P_{\Delta \nabla}\right),\left(P_{\nabla \Delta}\right),\left(P_{\Delta \Delta}\right)$, and $\left(P_{\nabla \nabla}\right)$, obtained from the direct discretizations of the continuous Euler-Lagrange equation (Section 4.1) and the discrete time-scale Euler-Lagrange equations (Section 4.2). All calculations were done using the Computer Algebra System Maple, version 10 (see Appendix $\mathrm{A}$ ). For problems $\left(P_{\Delta \Delta}\right)$ and $\left(P_{\nabla \nabla}\right)$ the discretization of the continuous Euler-Lagrange equation and the discrete time-scale Euler-Lagrange equations coincide. The Euler-Lagrange equation for problem $\left(P_{\Delta \Delta}\right)$ is defined on $\mathbb{T}^{\kappa^{2}}=\{0,1\}$ and we obtain a system of two equations with two unknowns $y_{1}$ and $y_{2}$ that leads to $y_{1}=2.322251304$ and $y_{2}=2.679109437$ with the cost functional value $K_{\Delta}(T) a_{\Delta}(T)=-16.97843026$. Similarly, the Euler-Lagrange equation for problem $\left(P_{\nabla \nabla}\right)$ is defined on $\mathbb{T}_{\kappa^{2}}=\{2,3\}$ and we obtain a system of two equations with two unknowns $y_{1}$ and $y_{2}$ that leads to $y_{1}=1.495415602$ and $y_{2}=2.228040364$ with the cost functional value $K_{\nabla}(T) a_{\nabla}(T)=-13.20842214$. As we show next, for hybrid deltanabla discrete problems of the calculus of variations, the time-scale results seem superior.

\subsection{Problem $\left(P_{\Delta \nabla}\right)$}

The Euler-Lagrange equations for problem $\left(P_{\Delta \nabla}\right)$ are defined on $\mathbb{T}_{\kappa}^{\kappa}=\{1,2\}$. Therefore, we obtain a system of equations with two unknowns $y_{1}$ and $y_{2}$. The discretized Euler-Lagrange equation $\left(E L_{P}\right)_{\Delta \nabla}$ gives

$$
y_{1}=2.910488556, \quad y_{2}=2.970017180
$$

with value of cost functional

$$
K_{\Delta}(T) a_{\nabla}(T)=-10.11399047 .
$$

A better result is obtained using the discrete time-scale Euler-Lagrange equation $E L_{P_{\Delta \nabla}}^{1}$ :

$$
y_{1}=2.901851949, \quad y_{2}=2.967442285
$$

with cost

$$
K_{\Delta}(T) a_{\nabla}(T)=-10.30544712
$$

\subsection{Problem $\left(P_{\nabla \Delta}\right)$}

The Euler-Lagrange equations for problem $\left(P_{\nabla \Delta}\right)$ are also defined on $\mathbb{T}_{\kappa}^{\kappa}=\{1,2\}$ and also lead to a system of two equations with the two unknowns $y_{1}$ and $y_{2}$. The discretized Euler-Lagrange equation $\left(E L_{P}\right)_{\nabla \Delta}$ gives

$$
y_{1}=2.183517532, \quad y_{2}=2.446990272
$$

with cost

$$
K_{\nabla}(T) a_{\Delta}(T)=-19.09167089 .
$$

Our time-scale Euler-Lagrange equation $E L_{P_{\nabla \Delta}}^{2}$ gives better results:

$$
y_{1}=2.186742579, \quad y_{2}=2.457402400
$$

with cost

$$
K_{\nabla}(T) a_{\Delta}(T)=-19.17699675 .
$$

The results are gathered in Table 1 . 


\begin{tabular}{|c|c|c|c|}
\hline \multirow{2}{*}{$D$} & \multicolumn{2}{|c|}{ The value of the functional of $\left(P_{D}\right), \rho=0.05$, for candidates to minimizers obtained from: } \\
\cline { 2 - 4 } & $\left(E L_{P}\right)_{D}$ & $E L_{P_{D}}^{\perp}$ & $E L_{P_{D}}^{2}$ \\
\hline$\Delta \nabla$ & -10.11399047 & -10.30544712 & $-0.1537986252 \times 10^{-5}$ \\
\hline$\nabla \Delta$ & -19.09167089 & 1020.105142 & -19.17699675 \\
\hline$\Delta \Delta$ & \multicolumn{3}{|c|}{-16.97843026} \\
\hline$\nabla \nabla$ & \multicolumn{3}{|c}{-13.20842214} \\
\hline
\end{tabular}

Table 1: The value of the functional associated to problem $P_{D}, D \in\{\Delta \nabla, \nabla \Delta, \Delta \Delta, \nabla \nabla\}$, with $\rho=0.05$, calculated using: (i) the direct discretization of the continuous Euler-Lagrange equation, that is, $\left(E L_{P}\right)_{D}$; (ii) discrete Euler-Lagrange equations $E L_{P_{D}}$, obtained from the calculus of variations on time scales with $\mathbb{T}=\mathbb{Z}$.

\section{Conclusion}

Some advantages of using the calculus of variations on time scales in economics were already discussed in [3, 5, 13. Here we considered two minimization discrete delta-nabla economic problems, denoted by $\left(P_{\Delta \nabla}\right)$ and $\left(P_{\nabla \Delta}\right)$, for which the time-scale approach leads to better results than the ones obtained by a direct discretization of the continuous necessary optimality condition: the approach on the right hand side of the diagram of Figure 1 gives candidates to minimizers for which the value of the functional is smaller than the values obtained from the approach on the left hand side of the diagram of Figure 1. It might be concluded that the time-scale theory of the calculus of variations leads to more precise results than the standard methods of discretization. For comparison purposes, we have used the same values for the parameters as the ones available in [11. We have, however, done simulations with other values of the parameters and the conclusion persists: in almost all cases the results obtained from our time-scale approach are better; hardly ever, they coincide with the classical method; never are worse. In particular, we changed the value of the discount rate, $\rho$, in the set $\{0.01,0.02,0.03, \ldots, 0.1\}$. This is motivated by the fact that this value depends much on the economic and politic situation. The case where the time-scale advantage is more visible is given in Table 2, which corresponds to a discount rate of $2 \%(\rho=0.02)$. The interested reader can easily do his/her own simulations using the Maple code found in Appendix A. For future work, we would like to generalize our mixed delta-nabla results, in particular Theorem 3.2 , for infinite horizon variational problems on time scales, that so far have been only studied in the delta 21] and nabla [14] cases.

\begin{tabular}{|c|c|c|c|}
\hline \multirow{2}{*}{$D$} & \multicolumn{2}{|c|}{ The value of the functional of $\left(P_{D}\right), \rho=0.02$, for candidates to minimizers obtained from: } \\
\cline { 2 - 5 } & $\left(E L_{P}\right)_{D}$ & $E L_{P_{D}}^{1}$ & $E L_{P_{D}}^{2}$ \\
\hline$\Delta \nabla$ & -10.62044023 & -10.70908681 & 0.00001078869584 \\
\hline$\nabla \Delta$ & -21.05128963 & $3.014255571 \times 10^{-8}$ & -264.5250742 \\
\hline$\Delta \Delta$ & \multicolumn{3}{|c|}{-19.035714446} \\
\hline$\nabla \nabla$ & \multicolumn{3}{|c|}{-14.19294557} \\
\hline
\end{tabular}

Table 2: The value of the functional associated to problem $P_{D}, D \in\{\Delta \nabla, \nabla \Delta, \Delta \Delta, \nabla \nabla\}$, with $\rho=0.02$, calculated using: (i) the direct discretization of the continuous Euler-Lagrange equation, that is, $\left(E L_{P}\right)_{D}$; (ii) discrete Euler-Lagrange equations $E L_{P_{D}}$, obtained from the calculus of variations on time scales with $\mathbb{T}=\mathbb{Z}$.

\section{A Maple Code}

We provide here all the definitions and computations done in Maple for the problems considered in Section 5. The definitions follow closely the notations introduced along the paper, and should be clear even for readers not familiar with the Computer Algebra System Maple. 


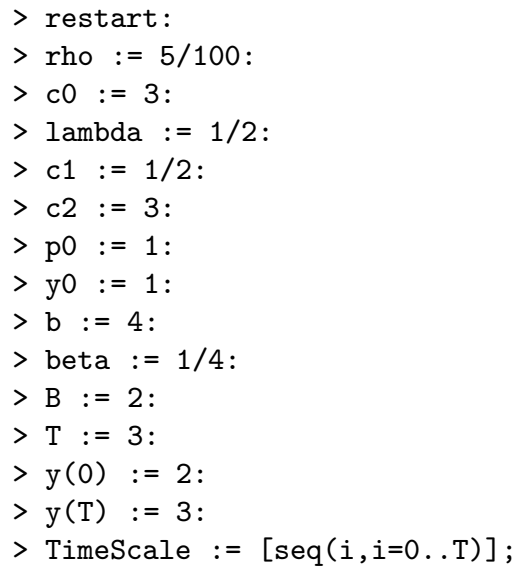

TimeScale $:=[0,1,2,3]$

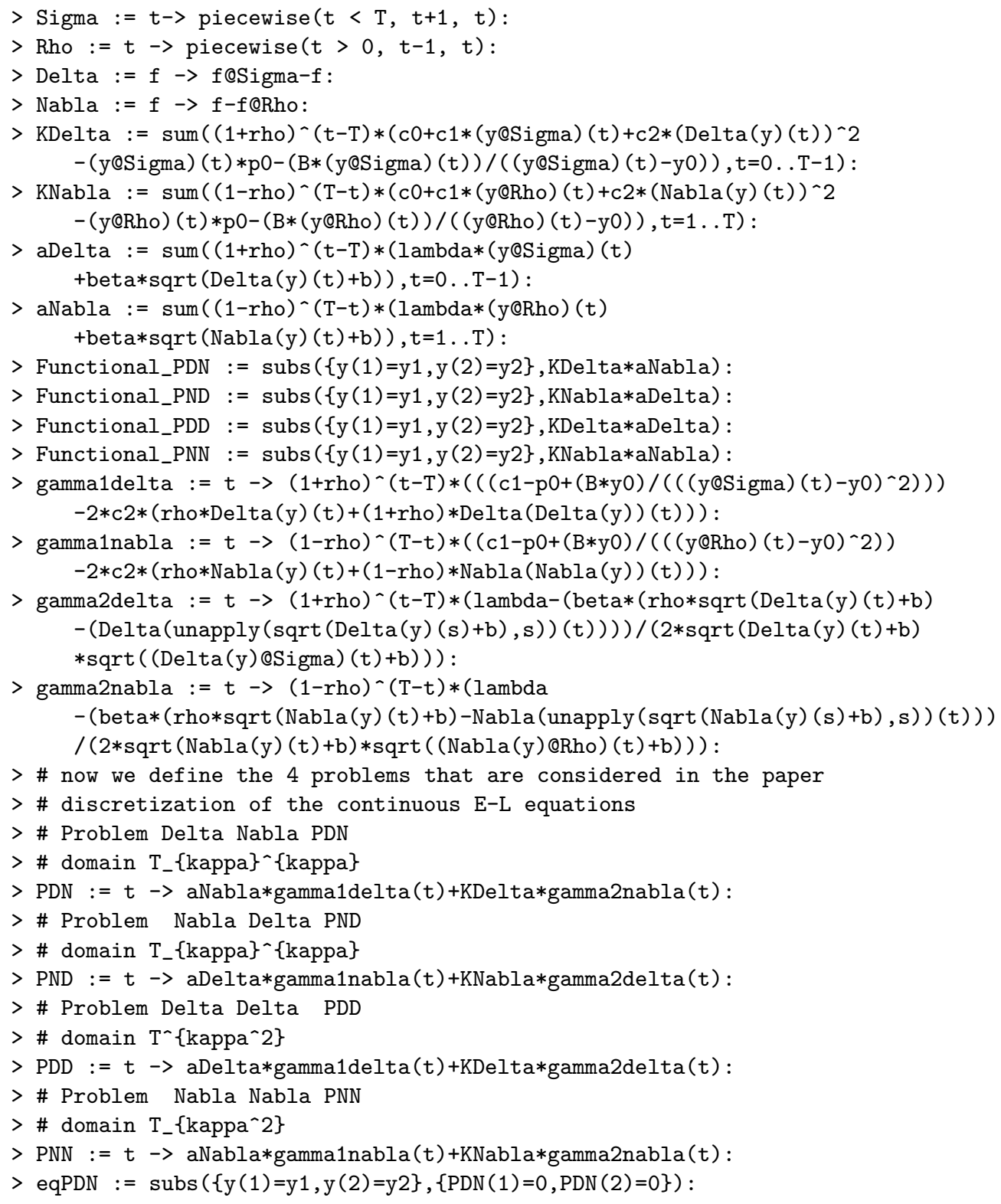




$$
\{y 1=0.5930298703, y 2=1.090438395\}
$$

subs (Solution_EL_eqs_system_nabla_version,Functional_PDN);

$>$ \# E-L equations for Problem Nabla Delta

$>\operatorname{part5}:=\mathrm{t} \rightarrow(1-\mathrm{rho})^{\wedge}(\mathrm{T}-\mathrm{Sigma}(\mathrm{t})) *\left(\mathrm{c} 1-\mathrm{p} 0+(\mathrm{B} * \mathrm{y} 0) /\left((\mathrm{y}(\mathrm{t})-\mathrm{y} 0)^{\wedge} 2\right)\right):$

$>$ part6 := t $\rightarrow 2 * c 2 *(1-r h o)^{\wedge}(\mathrm{T}-\operatorname{Sigma}(\mathrm{t})) *(\operatorname{rho} *(\operatorname{Nabla}(\mathrm{y})(\mathrm{t}))+(\operatorname{Delta}(\mathrm{Nabla}(\mathrm{y}))(\mathrm{t})))$ :

$>$ part7 $:=\mathrm{t} \rightarrow \operatorname{lambda} *(1+\mathrm{rho}) \wedge(\mathrm{Rho}(\mathrm{t})-\mathrm{T}):$

$>$ part8 $:=t \rightarrow(1+r h o) \wedge($ Rho $(t)-T) *((\operatorname{beta} *($ rho*sqrt $(\operatorname{Delta}(\mathrm{y})(\mathrm{t})+\mathrm{b})$

$-(1+\mathrm{rho}) * \mathrm{Nabla}($ unapply $(\operatorname{sqrt}(\operatorname{Delta}(\mathrm{y})(\mathrm{s})+\mathrm{b}), \mathrm{s}))(\mathrm{t})))$

$/(2 * \operatorname{sqrt}(\operatorname{Delta}(\mathrm{y})(\mathrm{t})+\mathrm{b}) * \operatorname{sqrt}(\mathrm{Nabla}(\mathrm{y})(\mathrm{t})+\mathrm{b}))):$

$>$ partDelta2 := Delta(unapply (aDelta*gamma1nabla $(t), t)$ ) @igma:

$>$ partNabla2 := Nabla (unapply (KNabla*gamma2delta $(t), t)$ )@Rho:

> \# E-L equation (10) for Problem Nabla Delta

$>$ EL_delta2 $:=t \rightarrow \mathrm{KNabla} *$ gamma2delta $(t)+\operatorname{aDelta} *(\operatorname{part5}(\mathrm{t})-\operatorname{part6}(\mathrm{t}))+\operatorname{partDelta2}(\mathrm{t}):$

> \#-L equation (11) for Problem Nabla Delta

$>$ EL_nabla2 $:=t \rightarrow \mathrm{KNabla} *(\operatorname{part7}(\mathrm{t})-\operatorname{part8}(\mathrm{t}))+\operatorname{aDelta} * g a m m a 1 n a b l a(t)-\operatorname{partNabla2}(\mathrm{t}):$

$>$ \# systems of E-L equations for Problem Nabla Delta

$>E_{-}$delta2_system $:=\operatorname{subs}\left(\{\mathrm{y}(1)=\mathrm{y} 1, \mathrm{y}(2)=\mathrm{y} 2\},\left\{\mathrm{EL}_{-} \operatorname{delta} 2(1)=0, \mathrm{EL}_{-} \operatorname{delta} 2(2)=0\right\}\right)$ :

$>$ Solution_EL_eqs_system_delta2_version := fsolve(EL_delta2_system, $\{\mathrm{y} 1, \mathrm{y} 2\})$;

Solution $_{E} L_{e} q s_{s} y_{\text {stem }}$ elta $2_{v}$ ersion $:=\{y 1=7.879260741, y 2=4.775003718\}$

$>\operatorname{subs}($ Solution_EL_eqs_system_delta2_version,Functional_PND) ;

1020.105142

$>$ EL_nabla2_system $:=\operatorname{subs}\left(\{\mathrm{y}(1)=\mathrm{y} 1, \mathrm{y}(2)=\mathrm{y} 2\},\left\{\mathrm{EL} \_n a b l a 2(1)=0, \mathrm{EL} \_n a b l a 2(2)=0\right\}\right)$ :

$>$ Solution_EL_eqs_system_nabla2_version := fsolve(EL_nabla2_system, $\{y 1, y 2\})$;

Solution $_{E} L_{e} q s_{s}$ ystem $_{n}$ abla $2_{v}$ ersion $:=\{y 1=2.186742579, y 2=2.457402400\}$

> subs(Solution_EL_eqs_system_nabla2_version,Functional_PND);

$-19.17699675$

\section{Acknowledgments}

This work was partially supported by Portuguese funds through the Center for Research and Development in Mathematics and Applications (CIDMA), and The Portuguese Foundation for Science and Technology (FCT), within project PEst-OE/MAT/UI4106/2014. Dryl was also supported by FCT through the Ph.D. fellowship SFRH/BD/51163/2010; Torres by FCT within project OCHERA, PTDC/EEI-AUT/1450/2012, co-financed by FEDER under POFC-QREN with COMPETE reference FCOMP-01-0124-FEDER-028894. The authors are very grateful to three anonymous referees, for several constructive remarks and suggestions.

\section{References}

[1] C. D. Ahlbrandt and C. Morian, Partial differential equations on time scales, J. Comput. Appl. Math. 141 (2002), no. 1-2, 35-55.

[2] D. Anderson, J. Bullock, L. Erbe, A. Peterson and H. Tran, Nabla dynamic equations, in Advances in dynamic equations on time scales, 47-83, Birkhäuser Boston, Boston, MA, 2003. 
[3] F. M. Atici, D. C. Biles and A. Lebedinsky, An application of time scales to economics, Math. Comput. Modelling 43 (2006), no. 7-8, 718-726.

[4] F. M. Atici and G. Sh. Guseinov, On Green's functions and positive solutions for boundary value problems on time scales, J. Comput. Appl. Math. 141 (2002), no. 1-2, 75-99.

[5] F. M. Atici and C. S. McMahan, A comparison in the theory of calculus of variations on time scales with an application to the Ramsey model, Nonlinear Dyn. Syst. Theory 9 (2009), no. $1,1-10$.

[6] M. Bohner, Calculus of variations on time scales, Dynam. Systems Appl. 13 (2004), no. 3-4, $339-349$.

[7] M. Bohner, G. Guseinov and A. Peterson, Introduction to the time scales calculus, in Advances in dynamic equations on time scales, 1-15, Birkhäuser Boston, Boston, MA, 2003.

[8] M. Bohner and A. Peterson, Dynamic equations on time scales, Birkhäuser Boston, Boston, MA, 2001.

[9] M. Bohner and A. Peterson, Advances in dynamic equations on time scales, Birkhäuser Boston, Boston, MA, 2003.

[10] M. C. Caputo, Time scales: from nabla calculus to delta calculus and vice versa via duality, Int. J. Difference Equ. 5 (2010), no. 1, 25-40. arXiv:0910.0085

[11] E. Castillo, A. Luceño and P. Pedregal, Composition functionals in calculus of variations. Application to products and quotients, Math. Models Methods Appl. Sci. 18 (2008), no. 1, $47-75$.

[12] J. Cresson, A. B. Malinowska and D. F. M. Torres, Time scale differential, integral, and variational embeddings of Lagrangian systems, Comput. Math. Appl. 64 (2012), no. 7, 22942301. arXiv: 1203.0264

[13] M. Dryl, A. B. Malinowska and D. F. M. Torres, A time-scale variational approach to inflation, unemployment and social loss, Control Cybernet. 42 (2013), no. 2, 399-418. arXiv: 1304.5269

[14] M. Dryl and D. F. M. Torres, Necessary optimality conditions for infinite horizon variational problems on time scales, Numer. Algebra Control Optim. 3 (2013), no. 1, 145-160. arXiv:1212.0988

[15] M. Dryl and D. F. M. Torres, The delta-nabla calculus of variations for composition functionals on time scales, Int. J. Difference Equ. 8 (2013), no. 1, 27-47. arXiv:1211.4368

[16] R. A. C. Ferreira and D. F. M. Torres, Remarks on the calculus of variations on time scales, Int. J. Ecol. Econ. Stat. 9 (2007), no. F07, 65-73. arXiv:0706.3152

[17] I. M. Gelfand and S. V. Fomin, Calculus of variations, Revised English edition translated and edited by Richard A. Silverman, Prentice Hall, Englewood Cliffs, NJ, 1963.

[18] E. Girejko, A. B. Malinowska and D. F. M. Torres, The contingent epiderivative and the calculus of variations on time scales, Optimization 61 (2012), no. 3, 251-264. arXiv:1007.0509

[19] E. Girejko and D. F. M. Torres, The existence of solutions for dynamic inclusions on time scales via duality, Appl. Math. Lett. 25 (2012), no. 11, 1632-1637. arXiv:1201.4495

[20] M. Gürses, G. Sh. Guseinov and B. Silindir, Integrable equations on time scales, J. Math. Phys. 46 (2005), no. 11, 113510, 22 pp. 
[21] A. B. Malinowska, N. Martins and D. F. M. Torres, Transversality conditions for infinite horizon variational problems on time scales, Optim. Lett. 5 (2011), no. 1, 41-53. arXiv:1003.3931

[22] A. B. Malinowska and D. F. M. Torres, The delta-nabla calculus of variations, Fasc. Math. 44 (2010), 75-83. arXiv:0912.0494

[23] A. B. Malinowska and D. F. M. Torres, Euler-Lagrange equations for composition functionals in calculus of variations on time scales, Discrete Contin. Dyn. Syst. 29 (2011), no. 2, 577-593. arXiv:1007.0584

[24] A. B. Malinowska and D. F. M. Torres, A general backwards calculus of variations via duality, Optim. Lett. 5 (2011), no. 4, 587-599. arXiv:1007.1679

[25] N. Martins and D. F. M. Torres, Calculus of variations on time scales with nabla derivatives, Nonlinear Anal. 71 (2009), no. 12, e763-e773. arXiv:0807.2596

[26] N. Martins and D. F. M. Torres, Necessary optimality conditions for higher-order infinite horizon variational problems on time scales, J. Optim. Theory Appl. 155 (2012), no. 2, 453476. arXiv:1204.3329

[27] H. Sagan, Introduction to the calculus of variations, Dover, New York, 1992.

[28] J. L. Troutman, Variational calculus and optimal control, second edition, Undergraduate Texts in Mathematics, Springer, New York, 1996. 